\title{
CRITICAL EXPONENT OF NEGATIVELY CURVED THREE MANIFOLDS
}

\author{
YONG HOU \\ Department of Mathematics, University of Iowa, Iowa City, Iowa 52242-1419, U.S.A. \\ e-mail:yhou@math.uiowa.edu
}

(Received 10 April, 2002; accepted 14 October, 2002)

\begin{abstract}
We prove that for a negatively pinched $\left(-b^{2} \leq \mathcal{K} \leq-1\right)$ topologically tame 3-manifold $\tilde{M} / \Gamma$, all geometrically infinite ends are simply degenerate. And if the limit set of $\Gamma$ is the entire boundary sphere at infinity, then the action of $\Gamma$ on the boundary sphere is ergodic with respect to harmonic measure, and the Poincare series diverges when the critical exponent is 2 .
\end{abstract}

2000 Mathematics Subject Classification. 57M50, 57M60.

1. Introduction. In the following $M$ is a complete Riemannian $n$-manifold with finitely generated fundamental group $\Gamma$. We assume that the sectional curvature $\mathcal{K}$ satisfies $-b^{2} \leq \mathcal{K} \leq-a^{2}$ for some $0<a \leq b$. A manifold which satisfies curvature bounds of this type will be said to have negatively pinched curvature. The Riemannian universal cover of $M$ is denoted by $\tilde{M}$, and $M$ is identified with $\tilde{M} / \Gamma$. The following additional notations and terminologies will also be used:

- $D$ is the critical exponent of the Poincare series $\left(\sum_{\gamma \in \Gamma} e^{-s \operatorname{dist}(x, \gamma x)}\right)$ of $\Gamma$. This means that for every $x \in \tilde{M}$, the series diverges when $s<D$ and converges when $s>D$.

- $\quad \Gamma$ is said to be divergent if the Poincaré series diverges at $s=D$.

- $\mathcal{S}$ denotes an arbitrary, fixed generating set of $\Gamma$.

- $\Lambda(\Gamma)$ is the limit set of $\Gamma$ (see Section 1$)$.

- For each $x \in \tilde{M}$ and $\gamma \in \Gamma$, we refer to $\operatorname{dist}(x, \gamma x)$ as the displacement of $x$ under $\gamma$.

- $\quad \Gamma$ is said to be harmonically ergodic if $\Gamma$ acts ergodically on $S_{\infty}$ with respect to the harmonic measure on $S_{\infty}$ (see Section 4).

- $\Gamma$ will be called topologically tame if $M=\tilde{M} / \Gamma$ is homeomorphic to the interior of a compact manifold-with-boundary.

THEOREM 1.1. Let $M$ be a topologically tame negatively pinched 3-manifold with $\Gamma$ purely loxodromic. Then all geometrically infinite ends of $M$ are simply degenerate.

THEOREM 1.2. Let $M=\tilde{M} / \Gamma$ be a topologically tame 3-manifold with $-b^{2} \leq$ $\mathcal{K} \leq-1$. Suppose that $\Gamma$ is purely loxodromic and that $\Lambda(\Gamma)=S_{\infty}$. Then $2 \leq D$ and $\bar{\Gamma}$ is harmonically ergodic. If $D=2$ then $\Gamma$ is also divergent.

The study of divergence of the Poincare series of the fundamental group of negatively curved manifolds is a crucial element in the investigation of geometric rigidity and displacement function estimates. One of the most fundamental tools in the theory of negatively curved manifolds is the Patterson-Sullivan conformal density supported on the limit set. It is a well-known theorem in hyperbolic geometry due to 
Sullivan, that a divergent Kleinian group is equivalent to the conical limit set of the group is of positive area. It was Shalen and Culler who first used Patterson-Sullivan density to study the displacement function of finitely generated non-elementary Kleinian groups, and subsequently proved that the least volume hyperbolic 3-manifold have Betti-number of most 2. Our main objective is to study geometric rigidity of infinite volume negatively pinched 3-manifolds (see [24]).

For hyperbolic 3-manifolds, Theorem 1.1 follows from a result of Canary's. In [8] Canary proved the geometrical tameness conjecture for topological tame hyperbolic 3-manifolds. The conjecture states that all finitely generated Kleinian groups are geometrically tame. It was the work of Bonahon which made the first breakthrough in this conjecture. For topologically tame hyperbolic 3-manifolds, Canary employed Bonahon's result [5] for the case where the compact core is boundary-irreducible and utilized the work of Gromov and Thurston [20] on branched coverings of hyperbolic manifolds to reduce the general case to the case of a boundary-irreducible compact core.

In section 2 we study some of the topological properties of negatively pinched 3-manifolds. In particular, Bonahon's theorem for negatively pinched 3-manifolds is stated. Section 3 is used to prove a stronger version of the Gromov-Thurston branched covering theorem. In Section 4, we prove Theorem 1.1 and deduce some immediate corollaries. Section 5 discusses measures on $S_{\infty}$ and the ergodicity of $\Gamma$ with respect to these measures.

2. Topological preliminary. In this section we will state some of the topological results that we will require for the proof of Theorem 1.1 in section 4 .

Every isometry of $\tilde{M}$ can be extended to a Lipschitz map on $S_{\infty}:=\partial \tilde{M}$ [19]. For a torsion-free $\Gamma$, every element $\gamma \in \Gamma$ is one of the following types: (1) parabolic if it has exactly one fixed point in $\tilde{M} \cup S_{\infty}$ which lies in $S_{\infty}$; (2) loxodromic if it has exactly two distinct fixed points in $\tilde{M} \cup S_{\infty}$, both lying in $S_{\infty}$.

Denote by $\Lambda(\Gamma) \subset \partial \tilde{M}$ the limit set of $\Gamma$, which is the unique minimal closed $\Gamma$-invariant subset of $S_{\infty}$. Most of the important properties of the limit set in the constant curvature space continue to hold in the variable curvature space [15]. In particular: (i) $\Lambda(\Gamma)=\overline{\Gamma x} \cap S_{\infty}$; (ii) $\Lambda(\Gamma)$ is the closure of the set of fixed points of loxodromic elements of $\Gamma$; and (iii) $\Lambda(\Gamma)$ is a perfect subset of $\Gamma$. The set $\Omega(\Gamma):=$ $S_{\infty} \backslash \Lambda(\Gamma)$ is the region of discontinuity. The action of $\Gamma$ on $\tilde{M} \cup \Omega(\Gamma)$ is proper and discontinuous, (see [15]). The manifold $M_{\Gamma}:=\tilde{M} \cup \Omega(\Gamma) / \Gamma$ with possibly nonempty boundary is traditionally called the Kleinian manifold. We also let $\Lambda_{c}(\Gamma)$ denote the conical limit set of $\Gamma$, i.e. $\xi \in \Lambda_{c}(\Gamma)$ if for some $x \in \tilde{M}$ (and hence for every $x$ ) there exist a sequence $\left(\gamma_{n}\right)$ of elements in $\Gamma$, a sequence $\left(t_{n}\right)$ of real numbers, and a real number $C>0$, such that $\gamma_{n} x \longrightarrow \xi$ and $\operatorname{dist}\left(c_{x}^{\xi}\left(t_{n}\right), \gamma_{n} x\right)<C$ where $c_{x}^{\xi}$ is the geodesic ray connecting $x$ and $\xi$. Equivalently, a point belongs to $\Lambda_{c}(\Gamma)$ if it belongs to infinitely many shadows cast by balls of some fixed radius centered at points of a fixed orbit of $\Gamma$. Note that $\Lambda_{c}(\Gamma)$ is a $\Gamma$-invariant subset of $\Lambda(\Gamma)$, and hence a dense subset.

Let Hull $(\Lambda(\Gamma))$ denote the convex hull of $\Lambda(\Gamma)$, which is the minimal $\Gamma$-invariant convex subset of $\tilde{M} \cup S_{\infty}$ containing the limit set. Then the convex set (Hull $(\Lambda(\Gamma))-$ $\Lambda(\Gamma)) / \Gamma$ is called the convex core of $M$. We denoted it by $\mathcal{C C}(M)$. Next we will recall a few facts about negatively curved 3-manifolds. The proofs can be found in [1].

Proposition 2.1 (Margulis Lemma). There exists a number $\epsilon_{b}$ which only depends on the pinching constant $b$ of $M$, such that the group $\Gamma_{\epsilon}$ generated by elements in $\Gamma$ of 
length at most $\epsilon_{b}$ with respect to a fixed point in $M$ is almost nilpotent of rank at most 2. Then the number $2 \epsilon_{b}$ is called the Margulis constant.

If $M$ is orientable and $\Gamma$ is torsion-free, then $\Gamma_{\epsilon_{b}}$ is abelian.

Let $\epsilon \leq \epsilon_{b}$ be given. Then $M$ may be written as the union of a thin part $M_{[0, \epsilon)}$ consisting of all points at which there is based a homotopically nontrivial loop of length $\leq \epsilon$ and a thick part $M_{[\epsilon, \infty)}=\overline{M-M_{[0, \epsilon)}}$. Note that $M_{[\epsilon, \infty)}$ is compact if $M$ is of finite volume. Also the thin part of $M$ is completely classified by the next proposition.

PROPOSITION 2.2. Each connected component of $M_{[0, \epsilon)}$ is diffeomorphic to one of the following:

parabolic rank-1 cusp: $S^{1} \times \mathbb{R} \times[0, \infty)$;

parabolic rank-2 cusp: $T^{2} \times[0, \infty)$;

solid torus about the axis of a loxodromic $\gamma: D^{2} \times S^{1}$.

For simplicity we restrict to the case where $M$ has no cusps. It follows from the existence of a compact core $C(M)$ for $M$ [14] that $M$ has only finitely many ends [2]. In fact, each component of $\partial C(M)$ is the boundary of a neighborhood of an end of $M$, and this gives a bijective correspondence between ends of $M$ and components of $\partial C(M)$.

Following Bonahon and Canary, we will define simplicial ruled surfaces as follows. Let $S$ be a surface of positive genus and let $T_{P}$ be a triangulation defined with respect to a finite collection $P$ of points of $S$. This means that $T_{P}$ is a maximal collection of nonisotopic essential arcs with end points in $P$; these arcs are the edges of the triangulation, and the components of the complement in $S$ of the union of the edges are the faces. Let $f: S \longrightarrow M$ be a map which takes edges to geodesic arcs and faces to nondegenerate geodesic ruled triangles in $M$. The map $f$ induces a singular metric on $S$. If the total angle about each vertex of $S$ with respect to this metric is at least $2 \pi$, then the pair $(S, f)$ is called a simplicial ruled surface. It follows from the definition of the induced metric on $S$ that $f$ preserves lengths of paths and is therefore distance non-increasing. Any geodesic ruled triangle in $M$ has Gaussian curvature at most $-a^{2}$. This means that each 2-simplex of $S$ inherits a Riemannian metric of curvature at most $-a^{2}$. Since we have required the total angle at each vertex to be at least $2 \pi$, by the Gauss-Bonnet theorem the curvature of $S$ is negative in the induced metric.

Definition 2.3. An end $E$ is said to be a geometrically infinite end if there exists a divergent sequence of geodesics, i.e: there exists a sequence of closed geodesics $\alpha_{k} \subset M_{\epsilon}^{\circ}$, such that for any neighborhood $U$ of $E$, there exists some positive integer $N$ such that $\alpha_{k} \subset U$ for all $k>N$. It follows from a result of Bonahon's, stated below as Proposition 1.5, that this definition of "geometrically infinite" is equivalent to a more intuitive definition. If in addition for some surface $S_{E}$ we have that $U$ is homeomorphic to $S_{E} \times[0, \infty)$, and there exists a sequence of simplicial ruled surfaces : $S_{E} \stackrel{f_{l}}{\longrightarrow} U$ such that $f_{l}\left(S_{E}\right)$ is homotopic to $S_{E} \times 0$ in $U$ and leaves every compact subset of $M$, then $E$ is said to be simply degenerate. The sequence $\left(S_{E} \stackrel{f_{l}}{\longrightarrow} U\right)$ is called an exiting sequence. An end which is not geometrically infinite will be called geometrically finite.

Thurston first defined simply degenerate ends by using pleated surfaces. Later Bonahon studied degenerate ends by using simplicial hyperbolic surfaces. In his thesis, Canary used simplicial ruled surfaces and studied the generalization of Bonahon's theorem to negatively pinched manifolds, which we will state next. 
THEOREM 2.4 (Bonahon; see [8]). Let $M=\tilde{M} / \Gamma$ be a negatively pinched complete Riemannian manifold with $\Gamma$ purely loxodromic. If $M$ has boundary-irreducible compact core, then every geometrically infinite end $E$ of $M$ is simply degenerate.

Bonahon's theorem will be essential for our proof of Theorem 1.1, which we will go through later. There are also a few simple but important results that were originally proved by Thurston and later reworked by Bonahon and Canary, which we will prove in our setting.

First, we state a characterization of geometric infiniteness by Bonahon [5], for the constant curvature case. However, the result continues to hold for manifolds of bounded negative curvature, with some straightforward modifications to the original proof.

Proposition 2.5 (Bonahon). If an end $E$ is not geometrically infinite, then there exists a neighborhood $U$ of $E$ which does not intersect $\mathcal{C C}(M)$.

COROLlaRY 2.6. If $M$ is noncompact with $\Gamma$ purely loxodromic and $\Lambda(\Gamma)=S_{\infty}$, then all ends are geometrically infinite.

Proof. Suppose there is a non-geometrically infinite end. By Proposition 2.5, there exists a neighborhood which is disjoint from $\mathcal{C C}(M)$, which implies that the region of discontinuity is nonempty, and hence contradicts the assumption that $\Lambda(\Gamma)=S_{\infty}$.

Proposition 2.7 (Bounded Diameter lemma). Let $(S, f)$ be a simplicial ruled surface such that for every compressible curve $\gamma$ on $S$, we have length $(f(\gamma)) \geq \epsilon$. Then for any $x, y \in S$ we have a path $\lambda$ in $S$ such that $f(\lambda) \cap M_{[\epsilon, \infty)}$ has length at most $\frac{8}{\epsilon} \pi a^{-2}|\chi(S)|$.

(Recall that $\epsilon$ is a positive number less than $\epsilon_{3, b}$. To say that $\gamma$ is compressible means that it represents a nontrivial element of the kernel of $f_{\#}: \pi_{1}(S) \rightarrow \pi_{1}(M)$.)

Proof. For $x, y$ in $S$, let $L_{x, y}$ denote the shortest curve connecting $x, y$. Denote $L_{x, y} \cap S_{[\epsilon, \infty)}$ by $L_{x, y}^{\epsilon}$. Then the $\epsilon / 4$-neighborhood $\left.N_{\epsilon / 4}\left(L^{\epsilon}\right)_{x, y}\right)$ of $L_{x, y}^{\epsilon}$ is an embedded tube in $S$. Since $(S, f)$ is a simplicial ruled surface, we have $\mathcal{K}(S) \leq-a^{2}$, where $S$ has the metric induced by $f$. The Gauss-Bonnet formula then gives

$$
\epsilon / 4 \text { length }\left(L_{x, y}^{\epsilon}\right) \leq \operatorname{area}\left(N_{\epsilon / 4}\left(L_{x, y}^{\epsilon}\right)\right) \leq 2 \pi a^{-2}|\chi(S)|,
$$

which implies that length $\left(L_{x, y}^{\epsilon}\right) \leq \frac{8}{\epsilon} \pi a^{-2}|\chi(S)|$. Also, by the assumption on the length of the image of compressible curve, and the fact that $f$ preserves lengths of paths, we have $f\left(S_{[0, \epsilon)}\right) \subset M_{[0, \epsilon)}$. Hence $f\left(L_{x, y}-L_{x, y}^{\epsilon}\right) \subset M_{[0, \epsilon)}$. Hence $f\left(L_{x, y}\right) \cap M_{[\epsilon, \infty]}$ is contained in $f\left(L^{\epsilon}\right)_{x, y}$, and therefore has length at most $\frac{8}{\epsilon} \pi a^{-2}|\chi(S)|$.

PROPOSITION 2.8. Let E be a simply degenerate end of $M$. Then there exists a number $\alpha>0$ and an exiting sequence $\left(S \stackrel{f_{k}}{\longrightarrow} U_{\alpha}\right)$ such that for any compressible curve $\gamma$ on $S$ we have length $\left(f_{k}(\gamma)\right) \geq \alpha$ for all $k$.

Proof. Let $\left(f_{l}\right)$ be any exiting sequence for $E$. Then there is a neighborhood $U=S_{E} \times[0, \infty)$ of $E$ such that $f_{l}(S) \subset U$ and $f_{l}: S \rightarrow U$ is a homotopy equivalence for every $l \geq 1$. Assume that there is a sequence $\left(\gamma_{l}\right)$ of compressing curves in $S$ such that length $\left(f_{l}\left(\gamma_{l}\right)\right.$ tends to 0 as $l \rightarrow \infty$. 
We claim that for each $l$ the curve $f_{l}\left(\gamma_{l}\right)$ is homotopic in $U$ to a closed curve $\eta_{l}$ such that length $\left(\eta_{l}\right) \leq$ length $\left(f_{l}\left(\gamma_{l}\right)\right)$ and $\eta_{l}$ meets $S_{E} \times\{0\}$. Since $f_{l}(\gamma)$ is homotopically trivial in $M$, it can be deformed to a constant through a continuous family of piecewise smooth curves whose lengths decrease monotonically. Now since $f_{l}\left(\gamma_{l}\right)$ is homotopically nontrivial in $U$, some curve in the family must meet the frontier $S_{E} \times\{0\}$ of $U$; the first such curve in the family is the required curve $\eta_{l}$.

In particular, length $\left(\eta_{l}\right)$ tends to 0 as $l \rightarrow \infty$. Hence for large enough $l$ we have $\eta_{l} \subset S_{E} \times[0,1]$. This gives a sequence of curves in the compact submanifold $S_{E} \times[0,1]$ of $M$ which are homotopically nontrivial in the submanifold, but whose lengths tend to 0 . This is a contradiction.

Proposition 2.9. Let $M=\tilde{M} / \Gamma$ be a negatively curved, topologically tame, orientable 3-manifold of infinite volume with purely loxodromic $\Gamma$. Let $\tilde{N}$ be a cover of $M$ such that $\pi_{1}(\tilde{N})$ is finitely generated. Then $\tilde{N}$ is also topologically tame.

Proof. By assumption, $M$ is topologically tame, so it is homeomorphic to the interior $N^{\circ}$ of a compact manifold $N$. Since $M$ is also negatively curved with infinite volume, so $N$ must be an irreducible, atoroidal manifold with nonempty boundary. Then by Thurston's uniformization theorem ([28], [30]), $N^{\circ}$ admits a geometrically finite, complete hyperbolic structure. Hence we can assume $N^{\circ}$ is a geometrically finite hyperbolic manifold. Now $\tilde{N}$ is homeomorphic to some covering space $\hat{N}$, a cover of $N^{\circ}$ with $\pi_{1}(\hat{N})$ finitely generated. Since $N^{\circ}$ is geometrically finite with infinite volume, $\hat{N}$ is also geometrically finite (this fact is proved by Thurston and presented in [28]), hence topologically tame. The result follows.

3. Deformation and branched cover. We will prove a branched cover theorem, stated below as Theorem 3.2, for 3-manifolds of variable negative curvature. This result will be used next for the proof of Theorem 1.1.

First we will point out a simple topological condition which will ensure the existence of a branched covering of a 3-manifold.

Proposition 3.1. Let p be a positive integer. Let $\gamma$ be a collection of null-homologous curves in a orientable 3-manifold $M$. Then there exists $a \mathbb{Z}_{p}$ cyclic branched covering of $M$ with $\gamma$ as the branching locus.

Let $N$ be a Riemannian $n$-manifold. We denote by $\mathcal{K}_{<a, b>}(N)$ the space of all metrics on $N$ which have negatively pinched curvature in the sense that $-b^{2} \leq \mathcal{K} \leq-a^{2}$.

THEOREM 3.2. Let $M$ be a 3-manifold and let $g$ be a metric in $g \in \mathcal{K}_{<a, b>}(M)$. Let $p$ be a positive integer. Let $\gamma$ be a collection of null-homologous simple closed geodesics in $(M, g)$. Then the $\mathbb{Z}_{p}$ branched covering $M_{p}$ of $M$ over the branch curves $\gamma$ admits $a$ metric $\bar{g}_{p}$ with $\bar{g}_{p} \in \mathcal{K}_{<a^{\prime}, b^{\prime}>}\left(M_{p}\right)$ for some $b^{\prime}>a^{\prime}>0$, and outside a neighborhood of the branch curves $\gamma$ the metric $\bar{g}_{p}$ is the pull-back metric of the metric $g$.

Theorem 3.2 will be proved at the end of this section. In Section 3.1 we will establish some geometric results which will be used in the proof of Theorem 3.2. In Section 3.2 the proof will be completed by combining these results with the following theorem:

THEOREM 3.3 (Gromov-Thurston [20]). Theorem 3.2 is true for a hyperbolic manifold $M$. 
3.1. Deformation of metric. Given a negatively pinched metric on a manifold and a simple closed curve in it, we will construct a new negatively pinched metric which, near the closed curve, is a hyperbolic metric.

Our construction is a generalization of the one given by Gao in [17]. In [17], a given metric with negative Ricci curvature is deformed into another negative Ricci curvature metric by local modification about a closed curve. This method is an important ingredient in the proof of a result due to Gao-Yau, which says that every closed 3-manifold $M$ admits a metric $g$ with $\operatorname{Ricc}(g)<0$, (see [18]).

Let $\left(N, g_{0}\right)$ be a smooth compact Riemannian $n$-manifold. Let $\mathcal{N}_{\rho}$ denote the radius $\rho$ tubular neighborhood of a simple closed oriented curve $\gamma$ in $N$. The radius $\rho$ is taken sufficiently small (i.e. $\rho<\operatorname{injrad} \gamma$ ) so that we can identify $\mathcal{N}_{\rho}$ topologically as the solid torus $D_{\rho}^{n-1} \times S^{1}$, where $D_{\rho}^{n-1}$ is the $n-1$ dimensional disc of radius $\rho$. We equip it with the cylindrical coordinates $(\theta, r, s)$ with $\theta \in \mathbb{R} / 2 \pi$. Let $\nabla$ be the Levi-Civita connection.

We denote the space of metrics on $N$ by $\mathcal{M}$. The space $\mathcal{M}$ can be made into a differentiable manifold with $T_{g} \mathcal{M}=\Gamma^{\infty}\left(S^{2} T^{*} N\right)$. Then the curvature operator $R$ : $\mathcal{M} \longrightarrow \Gamma^{\infty}\left(\Lambda^{2} T^{*} N \otimes \Lambda^{2} T^{*} N\right)$ is a quasilinear and differentiable operator (see [3] and [16]).

For a given tangent vector $h \in T_{g} \mathcal{M}$ we denote the derivative of $R$ at $g$ in the direction of $h$ by $\mathcal{D}_{h} R(g)$. By direct computation, one can show that for any $X_{i}, X_{j}, X_{k}, X_{l} \in \Gamma^{\infty}(T N)$ we have

$$
\begin{aligned}
\mathcal{D}_{h} R(g)\left(X_{i}, X_{j}, X_{k}, X_{l}\right)= & 1 / 2\left\{\nabla_{j k}^{2} h\left(X_{i}, X_{j}\right)+\nabla_{j l}^{2} h\left(X_{j}, X_{k}\right)-\nabla_{i k}^{2} h\left(X_{j}, X_{l}\right)\right. \\
& -\nabla_{j l}^{2} h\left(X_{i}, X_{j}\right)+h\left(R(g)\left(X_{i}, X_{j}\right) X_{k}, X_{l}\right) \\
& \left.-h\left(R(g)\left(X_{i}, X_{j}\right) X_{l}, X_{k}\right)\right\} .
\end{aligned}
$$

Here $\nabla_{i j}^{2} h:=\nabla_{i}\left(\nabla_{j} h\right)-\nabla_{i, j} h$ where $\nabla_{i, j}:=\nabla_{\nabla_{i} X_{j}}$, and $\nabla_{i j}^{2} h$ is the Hessian of $h$.

Now assume $h$ satisfies $h=\nabla h=0$ at $\gamma$. We will consider a deformation of $g$ the form $\tilde{g}:=g_{0}+t \psi h$, where $t$ ranges in $[0,1]$ and $\psi \in C^{\infty}(N,[0,1])$. Set $\tilde{h}:=\psi h$. Then, we have $\nabla_{i j}^{2} \tilde{h}=\psi \nabla_{i j}^{2} h+\mathcal{E}_{i j}$, where

$$
\mathcal{E}_{i j}:=D_{i j}^{2} \psi h+D_{j} \psi \nabla_{i} h+D_{i} \psi \nabla_{j} h-D_{i, j} \psi h
$$

Hence, we have

$$
\mathcal{D}_{\tilde{h}} R\left(g_{0}\right)\left(X_{i}, X_{j}, X_{k}, X_{l}\right)=\psi \mathcal{D}_{h} R\left(g_{0}\right)\left(X_{i}, X_{j}, X_{k}, X_{l}\right)+\mathcal{E}(\psi)\left(X_{i}, X_{j}, X_{k}, X_{l}\right)
$$

where

$$
\mathcal{E}(\psi)\left(X_{i}, X_{j}, X_{k}, X_{l}\right):=\frac{1}{2}\left(\mathcal{E}_{j k}\left(X_{i}, X_{l}\right)+\mathcal{E}_{i l}\left(X_{j}, X_{k}\right)-\mathcal{E}_{i k}\left(X_{j}, X_{l}\right)-\mathcal{E}_{j l}\left(X_{i}, X_{k}\right)\right) .
$$

Note that, since $h=\nabla h=0$ at $\gamma$, we have $\nabla_{i j}^{2} h\left(X_{k}, X_{l}\right)=D_{i j}^{2} h_{k l}$, where $h_{k l}:=h\left(X_{k}, X_{l}\right)$. So we get

$$
\left.\mathcal{D}_{h} R\left(g_{0}\right)\left(X_{i}, X_{j}, X_{k}, X_{l}\right)\right|_{\gamma}=\frac{1}{2}\left(\left(D_{j k}^{2} h_{i l}-D_{j l}^{2} h_{i k}\right)+\left(D_{i l}^{2} h_{j k}-D_{i k}^{2} h_{j l}\right)\right) .
$$

By the condition on the 1-jet of $h$ at $\gamma$ and $\left.\mathcal{E}(\psi)\right|_{\gamma}=0$, we have

$$
\left.R\left(g_{0}+t \tilde{h}\right)\right|_{\gamma}=\left.R\left(g_{0}+t h\right)\right|_{\gamma}=R\left(g_{0}\right)+t \psi \mathcal{D}_{h} R\left(g_{0}\right) .
$$


Hence if $g_{0}+t h$ defines a metric on $\mathcal{N}_{\rho}$ and $R\left(g_{0}\right)+t \psi \mathcal{D}_{h} R\left(g_{0}\right)$ is bounded in between $a^{2}$ and $b^{2}$ at $\gamma$, then it follows from continuity that for any small number $\delta>0$, there exists a $\tilde{\rho}<\rho$ such that the restriction of $g_{0}+t h$ to $\mathcal{N}_{\tilde{\rho}}$ is in $\mathcal{K}_{<a-\delta, b+\delta>}\left(\mathcal{N}_{\tilde{\rho}}\right)$. Note that the radius $\tilde{\rho}$ is independent of $\psi$ because $\psi$ is bounded by 1 . This is important if one is interested in deforming one metric to another. By (3), we have the operator equality $\mathcal{D}_{\tilde{h}}=\psi \mathcal{D}_{h}+\mathcal{E}(\psi)$. So if we consider the formal Taylor series $\sum \frac{\left(t \psi \mathcal{D}_{h}+t \mathcal{E}(\psi)\right)^{k}}{k !} R\left(g_{0}\right)$ we see that it can be made close to $\sum \frac{\left(t \mathcal{D}_{h}\right)^{k}}{k !} R\left(g_{0}\right)$, provided $\mathcal{E}(\psi)$ can be made sufficiently small, since $\psi$ has norm bounded by 1 . Hence, to ensure that $g_{0}+t \tilde{h}$ is also negatively pinched on $\mathcal{N}_{\tilde{\rho}}$, one needs to bound the term $\mathcal{E}(\psi)$ by a sufficiently small number, say $\epsilon>0$ on $\mathcal{N}_{\tilde{\rho}}$, with appropriate choice of $\psi$.

By (2), and since $h=\nabla h=0$ at $\gamma$, it is easy to see that, to bound $\mathcal{E}(\psi)$ by $\epsilon$, it would be sufficient to have $h D^{2} \psi$ and $\nabla h D \psi$ bounded by a constant multiple of $\epsilon$ on $\mathcal{N}_{\tilde{\rho}}$. We summarize these observations in the following proposition.

Proposition 3.4. Let $\gamma$ be a simple closed curve in $\left(N, g_{0}\right)$. Let $h \in \Gamma^{\infty}\left(S^{2} T^{*} N\right)$ satisfy $h=\nabla h=0$ at $\gamma$. Suppose that $\left(g_{0}+t h\right) \in \mathcal{K}_{<a, b>}\left(\mathcal{N}_{\tilde{\rho}}\right)$. Then for any given small $\delta>0$ less than a, there exists $\epsilon>0$, such that $\left(g_{0}+t \tilde{h}\right) \in \mathcal{K}_{<a-\delta, b+\delta>}\left(\mathcal{N}_{\tilde{\rho}}\right)$, provided that we have $\mathcal{E}(\psi)(W, X, Y, Z)<\epsilon$ for any unit vectors $W, X, Y, Z \in \Gamma^{\infty}(T N)$.

Next we will construct a family of functions indexed by $\epsilon>0$ which will satisfy the conditions of Proposition 3.4.

Let $\epsilon>0$ be any number. We choose a function $\beta_{\epsilon} \in C^{\infty}\left(\mathbb{R}^{+},[0,1]\right)$ such that $\beta_{\epsilon}((-\infty, \epsilon / 4])=0$ and $\beta_{\epsilon}([\epsilon / 3,+\infty))=1$. Set $c:=\max \left(\sup \left|\beta_{\epsilon}^{\prime}\right|, \sup \left|\beta_{\epsilon}^{\prime \prime}\right|\right)$ and $\bar{c}:=$ $\max (c, 1)$, and consider any $k$ such that $0<k<1 / \bar{c}$.

Let us define the function $\psi_{\epsilon} \in C^{\infty}([0, \rho],[0,1])$ to agree with $\beta_{\epsilon}\left(r^{k}\right)$ for $0 \leq r \leq$ $(3 \epsilon / 4)^{1 / k}$ and to be equal to 1 for $\rho \geq r \geq(3 \epsilon / 4)^{1 / k}$. Also let us denote the numbers; $(3 \epsilon / 4)^{1 / k},(2 \epsilon / 3)^{1 / k},(\epsilon / 4)^{1 / k}$ by $\bar{\rho}, \rho_{1}, \rho_{2}$ respectively.

Let $g_{0}, g_{1} \in \mathcal{K}_{<a, b>}\left(\mathcal{N}_{\rho}\right)$ be given. Set $h=g_{1}-g_{0}$.

Proposition 3.5. Let $\delta$ be a positive number, $\delta<a$. Suppose that $\left.h\right|_{\gamma}=\left.\nabla h\right|_{\gamma}=0$, and define a family of metrics $\left\{g_{\epsilon}\right\}_{\epsilon>0}$ on $\mathcal{N}_{\rho}$ by $g_{\epsilon}:=g_{0}+\psi_{\epsilon} h$. Then there exists a number $\epsilon(\delta)>0$ such that $g_{\epsilon(\delta)} \in \mathcal{K}_{<a-\delta, b+\delta>}\left(\mathcal{N}_{\bar{\rho}}\right)$ with $g_{\epsilon(\delta)}=g_{0}$ for $r \in\left[0, \rho_{2}\right)$ and $g_{\epsilon(\delta)}=g_{1}$ when $r \in\left(\rho_{1}, \bar{\rho}\right]$. (We will denote such $g_{\epsilon(\delta)}$ by $\left.\tilde{g}\right)$.

To prove Proposition 3.5 we will first compute $\mathcal{D}_{h} R\left(g_{0}\right)$ and verify that $R\left(g_{0}\right)+$ $t \psi_{\epsilon} \mathcal{D}_{h} R\left(g_{0}\right)$ is bounded between $a^{2}$ and $b^{2}$ at $\gamma$.

Let us extend the function $\psi_{\epsilon}$ to $\mathcal{N}_{\rho}$ trivially (i.e $\psi_{\epsilon}(\theta, r, s)=\psi_{\epsilon}(r)$ ). This will give us the deformation along the $r$ direction on $\mathcal{N}_{\tilde{\rho}}$. We will express $\mathcal{D}_{h} R\left(g_{0}\right)$ in local coordinates of $\mathcal{N}_{\rho}$. For a given metric $g$ we denote the Christoffel symbol and curvature of $g$ by $\Gamma_{j k}^{l}(g)$ and $R_{i j k l}$ respectively. A simple computation gives us the following;

$$
\left.R_{i j k l}\left(g_{1}\right)\right|_{\gamma}-\left.R_{i j k l}\left(g_{0}\right)\right|_{\gamma}=\left(\partial_{i k}^{2} h_{j l}-\partial_{i l}^{2} h_{j k}\right)+\left(\partial_{j l}^{2} h_{i k}-\partial_{j k}^{2} h_{i l}\right)
$$

Comparing this with equation (4), we have:

$$
\left.\left(\mathcal{D}_{h} R\left(g_{0}\right)\right)_{i j k l}\right|_{\gamma}=\left.R_{i j k l}\left(g_{1}\right)\right|_{\gamma}-\left.R_{i j k l}\left(g_{0}\right)\right|_{\gamma} .
$$

Then by equation (5), we have $\left.R(\hat{g})\right|_{\gamma}=R\left(g_{0}\right)+t \mathcal{D}_{h} R\left(g_{0}\right)$. So, for $t \in[0,1]$, we get $a^{2} \leq R(\hat{g}) \leq b^{2}$. Therefore, for any given small $\delta>0$, by continuity, there exists $\tilde{\rho}>0$ such that $\hat{g}$ is in $\mathcal{K}_{<a-\delta, b+\delta>}\left(\mathcal{N}_{\tilde{\rho}}\right)$. 
Proof of Proposition 3.5. By Proposition 3.4, we only need to show that $h D^{2} \psi_{\epsilon}$ and $\nabla h D \psi_{\epsilon}$, which in local coordinates are $\partial_{i} \psi_{\epsilon} \partial_{j} h_{k l}$ and $h_{i j} \partial_{k l}^{2} \psi_{\epsilon}$ respectively, are bounded by a constant multiple of $\epsilon$.

Since $h=\nabla h=0$ at $\gamma$, we have

$$
h_{k l}=\sum_{i, j} c_{i k j l} x_{i} x_{j}+O\left(|x|^{3}\right) \quad \text { locally around } \gamma,
$$

where $\left\{x_{l}\right\}$ denotes the local coordinates $(\theta, r, s)$. So the above equation implies that there exist $c, c^{\prime}>0$ such that $\left|h_{i j}\right| \leq c|x|^{2}$ and $\left|\partial_{j} h_{k l}\right| \leq c^{\prime}|x|$ on the compact set $D^{n-1} \times$ $S^{1}$. Hence we only need to bound terms $|x|\left|\partial_{i} \psi_{\epsilon}\right|$ and $|x|^{2}\left|\partial_{k l} \psi_{\epsilon}\right|$ by a constant multiple of $\epsilon$. Since the function $\psi_{\epsilon}$ depends only on $r=|x|$, we may rewrite $|x|\left|\partial_{i} \psi_{\epsilon}\right|$ and $|x|^{2}\left|\partial_{k l} \psi_{\epsilon}\right|$ simply as $r\left|\psi_{\epsilon}^{\prime}\right|$ and $r^{2}\left|\psi_{\epsilon}^{\prime \prime}\right|$, where ' denotes differentiation with respect to $r$.

We may assume $\epsilon<\min (\tilde{\rho}, 1)$. Then it is easy to verify that $\max \left(r\left|\psi_{\epsilon}^{\prime}\right|, r^{2}\left|\psi_{\epsilon}^{\prime \prime}\right|\right)<$ $2 \epsilon$, and $0<\rho_{2}<\rho_{1}<\bar{\rho}<\tilde{\rho}$. Since $\tilde{g}$ satisfies

$$
\tilde{g}=\left\{\begin{array}{l}
g_{0}: 0 \leq r \leq \rho_{2} \\
g_{1}: \rho_{1} \leq r \leq \bar{\rho}
\end{array}\right.
$$

and $\epsilon>0$ is arbitrarily small, the result follows from Proposition 3.4.

3.2. Branched covers. Let $(M, g)$ be a Riemannian 3-manifold with $-b^{2} \leq$ $\mathcal{K} \leq-a^{2}$. Let $\gamma$ be a simple closed geodesic parametrized by arclength in $M$. Let $\tilde{\gamma}$ be a lift of $\gamma$ in $\tilde{M}$. Then $\tilde{\gamma}$ is an axis of some loxodromic element $\alpha$ in $\Gamma$. The restriction of $\alpha$ to the axis $\tilde{\gamma}(s)$ is translation through some distance $l$. The distance $l$ is called the length of $\alpha$. With the appropriate parametrization we can write $\alpha(\tilde{\gamma}(s))=\tilde{\gamma}(s+l)$.

We equip $\mathcal{N}_{\rho}(\tilde{\gamma})$ with a hyperbolic metric:

$$
\tilde{f}=\mathrm{d} r^{2}+\sinh ^{2}(r) \mathrm{d} \theta^{2}+\cosh ^{2}(r) \mathrm{d} s^{2}
$$

with respect to the cylindrical coordinates $(r, \theta, s)$ on $\mathcal{N}_{\rho}(\tilde{\gamma})$, where $s$ denotes arclength. The identity component of the isometry group for $\tilde{f}$ is $S O(2) \times T ; T$ is the group of translations along $\tilde{\gamma}$. Let $\partial_{s}:=\dot{\gamma}(s)$ denote the parallel vector field along $\gamma$. Then $\mathcal{N}_{\rho}(\gamma)=\exp _{\gamma}\left(\partial_{s} \times \partial_{s}^{\perp}\right)$ for $\rho \leq \operatorname{injrad} \gamma$. Let $\left\{u_{1}(s), u_{2}(s)\right\}$ be an orthonormal frame along $\gamma$ which spans $\partial_{s}^{\perp}$, with $u_{1}(0)=\partial_{r}, u_{2}(0)=\partial_{\theta}$. Consider the smooth map $F:[0, l] \times D_{\rho}^{2} \longrightarrow \mathcal{N}_{\rho}(\gamma)$ defined by:

$$
F\left(s, x^{1}, x^{2}\right)=\exp _{\gamma(s)}\left(\sum_{i} x^{i} u_{i}\right) .
$$

The map $F$ defines a system of coordinate on $\mathcal{N}_{\rho}(\gamma)$, called the Fermi coordinates.

Parallel transport around $\gamma$ gives a rotation $\omega \in \mathrm{SO}(2)$. We have:

Proposition 3.6. Let $f$ be the metric implicitly defined by $\tilde{f}=\pi_{1}^{*} f$, where $\pi_{1}$ is the projection map: $D_{\rho}^{2} \times \mathbb{R} \longrightarrow D_{\rho}^{2} \times \mathbb{R} /\left(\omega \times T_{l}\right)$. Define $h:=f-g$. Then $h=\nabla h=0$ at $\gamma$.

Proof. By using Fermi coordinates and the fact that $\nabla$ is the Levi-Civita connection we have $\left.g\right|_{\gamma}=\mathrm{d} s^{2}$ and $\left.\nabla g\right|_{\gamma}=0$. Also, from the formula for $\tilde{f}$, we see that $\left.\partial_{i} \tilde{f_{j k}}\right|_{r=0}=0$, so $\left.\nabla f\right|_{\gamma}=0$. Hence we have $h=\nabla h=0$ on $\gamma$. 
Proof of Theorem 3.2. Let $\tilde{M}_{p} \stackrel{\tilde{\pi}_{p}}{\longrightarrow} \tilde{M}$ be the $\mathbb{Z}_{p}$ cyclic branched covering of $\tilde{M}$ with branching curve $\gamma$. By Propositions 3.5 and 3.6 we have two numbers $\rho_{1}>0$, $\rho_{2}>0$ with $\rho_{1}<\rho_{2}<\rho$, and a metric $\bar{g}$ on $M$ such that

$$
\bar{g}=\left\{\begin{array}{l}
f: r \leq \rho_{2} \\
g: r \geq \rho_{1} .
\end{array}\right.
$$

Let $\tilde{\bar{g}}$ be the lift of $\bar{g}$ to $\tilde{M}$. Then, on the complement of a neighborhood $\tilde{\pi}_{p}^{-1} \mathcal{N}_{\rho^{\prime}}(\tilde{\gamma})$ of $\tilde{\gamma}$ in $\tilde{M}_{p}$ where $0<\rho^{\prime} \ll \rho_{2}$, the pullback metric of $\tilde{\bar{g}}$ is of the form:

$$
\mathrm{d}^{2} r+p^{2} \sinh (r)^{2} \mathrm{~d}^{2} \theta+\cosh (r)^{2} \mathrm{~d}^{2} s
$$

for $\rho^{\prime} \leq r \leq \rho_{2}$, and agrees with $\tilde{\pi}_{p}^{*} \tilde{g}$ for $\rho_{1} \leq r$.

Next, we simply point out that according to [20, Lemma 2.1], there exists a $C^{\infty}$ function $\sigma(r)$, which is equal to $\sinh (r)$ for $0 \leq r \ll \rho^{\prime}$ and is equal to $p \sinh (r)$ for $r \geq \rho^{\prime}$, and satisfies the following inequalities:

$$
\begin{gathered}
\sigma^{\prime}, \sigma^{\prime \prime}>0 \\
\lambda^{-1} \leq \sigma^{\prime \prime} / \sigma \leq \lambda \\
\lambda^{-1} \leq \frac{\sigma^{\prime} \sinh (r)}{\sigma \cosh (r)} \leq \lambda
\end{gathered}
$$

for some $\lambda>0$ which only depends on $p$ and $\rho^{\prime}$.

We can use $\sigma$ to define a $C^{\infty}$ metric $\widetilde{\bar{g}}_{p}$ on $\tilde{M}_{p}$ which is in $\mathcal{K}_{<a^{\prime}, b^{\prime}>}\left(\tilde{M}_{p}\right)$. Explicitly, on the neighborhood of $\tilde{\gamma}$ defined by $0 \leq r \leq \rho_{2}$, the metric $\widetilde{\bar{g}_{p}}$ is just

$$
\mathrm{d}^{2} r+\sigma(r)^{2} \mathrm{~d}^{2} \theta+\cosh (r)^{2} \mathrm{~d}^{2} s
$$

On the complement of this neighborhood, $\widetilde{\bar{g}_{p}}$ agrees with $\tilde{\bar{g}}$.

Finally, it is easy to see from the construction that $\widetilde{\bar{g}}_{p}$ is invariant under all isometries of the cover $\tilde{M}_{p}$. Hence we have a metric $\bar{g}_{p}$ on $M_{p}$ with $\widetilde{\bar{g}}_{p}=\pi_{2}^{*} \bar{g}_{p}$, where $\pi_{2}^{*}$ is given by the commutative diagram:

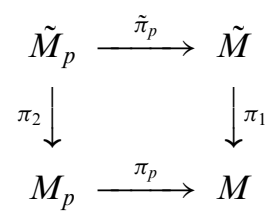

\section{Ends.}

THEOREM 4.1. Let $M$ be a topologically tame negatively pinched 3-manifold with $\Gamma$ purely loxodromic. Then all geometrically infinite ends of $M$ are simply degenerate.

The strategy of Canary's proof ([8, Proposition 5.1]) of geometric tameness for topologically tame hyperbolic manifolds works equally well for manifolds of variable negative curvature. Hence we will summarize the steps involved in Canary's proof and mention the necessary modifications that one will need for the proof of Theorem 4.1. 
Proposition 4.2. Let $M$ be a complete, topologically tame, negatively pinched 3manifold, with an end $E$. Then there exists a complete, negatively pinched 3-manifold $M^{\prime}$ with an end $E^{\prime}$ such that (i) some neighborhood $U_{E^{\prime}}$ of $E^{\prime}$ is isometric to a neighborhood $U_{E}$ of $E$, and (ii) the compact core of $M^{\prime}$ is boundary-irreducible.

Proof. By topological tameness, $M$ is homeomorphic to the interior of a compact 3-manifold $N$. The manifold $N$ can be decomposed into a finite union of compression bodies and boundary irreducible manifolds [22]. Then $E$ is associated with a boundary component of a compression body $C$. Note that by Proposition 2.9 a cover of $M$ with finitely generated fundamental group is also topologically tame. The Riemannian cover of $M$ associated to $\pi_{1}(C)$, has an end isometric to $E$. Hence after replacing $M$ by this Riemannian cover, we may assume that $M$ is homeomorphically identified with the interior of $C$. The idea then is to show that there is an collection of curves $\gamma$ such that the $\mathbb{Z}_{2}$ cyclic branched cover $C_{b}$ of $C$ over $\gamma$ has incompressible boundary. The construction of $C_{b}$ is based on the fact (which is established in [8] as a consequence of the Meeks-Yau Equivariant Dehn's lemma [27]) that if $\gamma$ is a disjoint union of simple closed curves that intersects every essential disk in $C$, then any 2-fold cyclic branched cover of $C$ with branch locus $\gamma$ is boundary-irreducible. Hence, in view of Proposition 2.1, the problem reduces to showing that there exists a collection of null-homologous simple geodesics $\gamma$ that intersects each essential disk.

Otal's theorem $[\mathbf{8}, 3.5]$ provides a disjoint union $\gamma_{0}$ of simple closed curves in $M=C^{\circ}$ which intersects every essential disk in $C$. Let $\gamma_{1}$ denote the collection of closed geodesics in $M$ with homeomorphic images in $C$ which are homotopic to $\gamma_{0}$. The geodesics $\gamma_{1}$ need not be simple; however, by using the same technique as in [8, Lemma 5.5] we may perturb the metric of $M$ in a compact neighborhood of $\gamma_{1}$ so as to obtain a metric which is also negatively pinched, and such that the geodesics with respect to the new metric which represent the same homotopy classes as $\gamma_{0}$ form a disjoint family of simple geodesics. Therefore, without loss of generality, we may assume that $\gamma$ is a collection of simple closed geodesics. The 2-fold cyclic branched cover of $C$ over $\gamma$ is then the desired $C_{b}$. We define $M^{\prime}$ to be the interior of $C_{b}$, which is a 2 -fold branched cover of $M$ with branch locus $\gamma$.

Next we note that by Theorem 3.2, $M^{\prime}$ can be given an metric with negatively pinched curvature with pinching constants arbitrarily close to the pinching constant of $M$ and outside a compact tubular neighborhood of the branching geodesics, the metric is induced by the metric on $M$ via the branched covering map, and hence its ends are isometric to the ends of $M$. Therefore the result follows.

Proof of Theorem 4.1. The result follows immediately from Proposition 4.2 and Theorem 2.4.

4.1. Corollaries. In this section we deduce two consequences of Theorem 4.1, Corollaries 4.4 and 4.7, which are of special interest. They relate topological tameness, which is a purely topological condition, to an analytical property. This interaction between topology and analysis was originally worked out for hyperbolic manifolds by Thurston in the case of a boundary-irreducible compact core, and generalized by Canary, Culler and Shalen to cases where the core may be boundary reducible. However the idea works equally well for variable curvature manifolds, as we shall now show. 
Definition 4.3. An orientable Riemannian manifold $N$ (with possibly non-empty boundary) is analytically tame if there exists an sequence of compact submanifolds $\left(N_{k}\right)$ such that

(i) $N_{k_{1}} \subset N_{k_{2}}$ for $k_{1}<k_{2}$;

(ii) if $N_{k}^{\circ}$ denotes the interior of $N_{k}$, then $\cup_{k} N_{k}^{\circ}=N$;

(iii) there exists a number $C>0$ such that for some $r>0$ we have, for all $k$,

$$
\operatorname{volume}\left(\mathcal{N}_{r}\left(\partial N_{k}\right)\right) \leq C .
$$

Such a sequence $\left(N_{k}\right)$ is called an exhaustive sequence.

COROLlARY 4.4. Let $M=\tilde{M} / \Gamma$ be a topologically tame negatively pinched $\left(-b^{2} \leq\right.$ $\left.\mathcal{K}(M) \leq-a^{2}\right)$ 3-manifold with $\Gamma$ purely loxodromic. Then $\mathcal{C C}(M)$ is analytically tame.

Proof. First we note that, under the hypothesis, there are no cusps. Hence the ends of $\mathcal{C C}(M)$ are precisely the geometrically infinite ends of $M$. By Theorem 4.1, these ends are simply degenerate.

For each geometrically infinite end $E$ of $M$, let $U_{E}$ be an neighborhood of $E$, which is homeomorphic to $S_{E} \times[0, \infty)$, and choose a sequence of maps $\left(f_{k}^{E}: S_{E} \rightarrow U_{E}\right)$ having the properties stated in the definition of a simply degenerate end. By passing to a subsequence of $\left(f_{k}^{E}\right)$, we may assume that the $f_{k}^{E}$ satisfy the following property (1): $f_{k}^{E}\left(S_{E}\right) \cap f_{l}^{E}\left(S_{E}\right)=\emptyset$ for $k \neq l$, and $f_{i}^{E}\left(S_{E}\right)$ is enclosed by $\left(f_{j}^{E}\left(S_{E}\right) \cup \partial U_{E}\right)$ for $i<j$, i.e. $f_{i}^{E}\left(S_{E}\right)$ is contained in the relatively compact subset of $U_{E}$ with boundary $f_{j}^{E}\left(S_{E}\right) \cup \partial U_{E}$. Furthermore, Proposition 2.8, applied to each of the finitely many geometrically infinite ends of $M$, gives a constant $\alpha>0$ such that for geometrically infinite end $E$ and any compressible curve $C$ in $S_{E}$, we have (2): length $\left(f_{k}^{E}(C)\right)>\alpha$. Then for each $k$ we define a compact submanifold $M_{k}$ to be the region in $M$ enclosed by $\partial \mathcal{C C}(M) \cup \bigcup_{E} f_{k}^{E}\left(S_{E}\right)$, where $E$ ranges over all geometrically infinite ends of $M$.

We verify that $\left(M_{k}\right)$ is the desired exhaustive sequence for $\mathcal{C C}(M)$. By the distancenonincreasing property of the maps $f_{k}^{E}$, together with property (2) above, one can show that (compare [8, Lemma 8.2])

$$
\begin{aligned}
\operatorname{volume}\left(\mathcal{N}_{r}\left(f_{k}\left(S_{E}\right)\right)\right) & \leq \sum_{E} \frac{1}{d(\alpha / 2)} \int_{S_{E}} B(r+(\alpha / 2)) \mathrm{d} A \\
& \leq \sum_{E} \frac{2 a^{-2} \pi B(r+(\alpha / 2))\left|\chi\left(S_{E}\right)\right|}{d(\alpha / 2)}
\end{aligned}
$$

where $d(\alpha / 2)$ is the least area of any disc of radius $\alpha / 2$ in the universal cover $\tilde{S}_{E}$ of $S_{E}$, and $B(r+(\alpha / 2))$ is the greatest volume of any ball of radius $r+\alpha / 2$ in $\tilde{M}$. If $V^{n}(r)$ denotes the volume of a ball of radius $r$ in $\mathbb{H}^{n}$, we have $B(r+\alpha / 2) \leq c_{b} V^{3}(r+\alpha / 2)$ for some constant $c_{b}$ depending only on $b$, and $d(\alpha / 2) \geq c_{a} V^{2}(\alpha / 2)$ for some constants $c_{b}$ and $c_{a}$ depending only on $b$ and $a$ respectively. Hence as $k \rightarrow \infty$ the quantity $\operatorname{volume}\left(\mathcal{N}_{r}\left(\partial M_{k}\right)\right)$ is bounded by some positive constant $C=C(M, r)$. And the assertions that $\cup_{k} M_{k}^{\circ}=\mathcal{C C}(M)$ and $M_{k_{1}} \subset M_{k_{2}}$ for $k_{1}<k_{2}$ follow directly from the definition of $M_{k}$. This completes the proof.

THEOREM 4.5 (compare $[\mathbf{8}, 35]$ ). Let $N$ be a complete analytically tame manifold. Then, any subharmonic function $u$ which is bounded above on $N$ is constant.

Proof. Assume that there exists a nontrivial subharmonic function bounded above on $N$, and denote it by $u$. Let $\lambda>0$ be such that $u \leq \lambda$. By normalization we can 
assume $\lambda=1$. Let $g_{t}$ denote the flow of the vector field $\operatorname{grad} u$. Note that $g_{t}$ is defined for all $t \geq 0$, and for any measurable subset $A \subset N$ we have

$$
\begin{aligned}
\left.\frac{\mathrm{d}}{\mathrm{d} t} \operatorname{volume}\left(g_{t}(A)\right)\right|_{t=s} & =\left.\int_{A} \frac{\mathrm{d}}{\mathrm{d} t} g_{t}^{*} \mathrm{~d} v\right|_{t=s}=\left.\int_{A} g_{s}^{*} \frac{\mathrm{d}}{\mathrm{d} t} g_{t}^{*} \mathrm{~d} v\right|_{t=0} \\
& =\int_{A} g_{s}^{*} L_{\operatorname{grad} u} \mathrm{~d} v=\int_{A} g_{s}^{*}((\operatorname{div}(\operatorname{grad} u)) \mathrm{d} v) \\
& =\int_{A} g_{s}^{*}(\Delta u \mathrm{~d} v)=\int_{g_{s}(A)} \Delta u \mathrm{~d} v \\
& \geq 0
\end{aligned}
$$

which means that $g_{t}$ is volume nondecreasing. Let $x$ be any point of $N$, let $B$ be any subset of $\left\{g_{t}(x)\right\}_{t \geq 0}$ and $T(B)$ denote the amount of time that $g_{t}(x) \in B$. Then, we have

$$
\begin{aligned}
(\text { length }(B))^{2} & =\left(\int_{0}^{T(B)}\left\langle\frac{\mathrm{d} g_{t}}{\mathrm{~d} t}, \frac{\mathrm{d} g_{t}}{\mathrm{~d} t}\right\rangle^{1 / 2} \mathrm{~d} t\right)^{2} \leq T(B) \int_{0}^{T(B)}\left\langle\frac{\mathrm{d} g_{t}}{\mathrm{~d} t}, \frac{\mathrm{d} g_{t}}{\mathrm{~d} t}\right\rangle \mathrm{d} t \\
& \leq T(B) \int_{0}^{T(B)}\left\langle\operatorname{grad} u\left(g_{t}(x)\right), \operatorname{grad} u\left(g_{t}(x)\right)\right\rangle \mathrm{d} t \\
& \leq T(B) \int_{0}^{T(B)}(\operatorname{grad} u)\left(u\left(g_{t}\right)\right)(x) \mathrm{d} t \\
& \leq T(B) \int_{0}^{T(B)} \mathrm{dg}_{t}^{*}(u)(x) \leq T(B)
\end{aligned}
$$

where the last inequality follows from our assumption that $u$ is bounded above by 1 .

We wish to show that $u$ is constant. Assume it is not, and let $x$ be a point of $M$ such that $\operatorname{grad} u(x) \neq 0$. Let $V$ be a neighborhood of $x$ in $N$ of positive volume with $V \cap g_{\tau}(V)=\emptyset$ for some $\tau>0$. Note that such a set $V$ can always be found since $u$ is strictly increasing along the flow line near $x$. Now, if $V \cap g_{\tau}(V)=\emptyset$, then $g_{n \tau}(V) \cap g_{m \tau}(V)=\emptyset$ for any positive integers $n$ and $m$, because $g_{t}$ is a flow . Let $\left\{N_{k}\right\}$ be the exhaustive sequence of $N$. Since $g_{t}$ is volume nondecreasing and $N_{k}$ is compact, we must have $g_{T_{k}}(V) \subset N-N_{k}$ for some large finite $T_{k}$. Now, let $V_{1}$ be any subset of $V$ of positive volume with diameter $g_{t}\left(V_{1}\right)<1$ for $t \leq T_{k}$. Note that for some small $r>0$ we have that $\mathcal{N}_{r}\left(\partial N_{k}\right) \cap \mathcal{N}_{r}\left(\partial N_{k+1}\right)=\emptyset$. By passing to a subsequence if necessary, we can take $r=1$. Then for any $y \in V_{1}$, the flow line $\left\{g_{t}(y)\right\}$ that passes through $\cup_{0}^{k} \mathcal{N}_{1}\left(\partial N_{k}\right)$ will have length at least $2 k$, since $\mathcal{N}_{1}\left(\partial N_{i}\right) \cap \mathcal{N}_{1}\left(\partial N_{i+1}\right)=\emptyset$. So the amount of time that $g_{t}(y)$ spends in $\cup_{0}^{k} \mathcal{N}_{1}\left(\partial N_{k}\right)$ is at least $(2 k)^{2}$. Hence $g_{l}\left(V_{1}\right) \subset$ $\cup_{0}^{k} \mathcal{N}_{2}\left(\partial N_{k}\right)$ for all $0 \leq l \leq 4 k^{2}$. Since $g_{l}\left(V_{1}\right) \cap g_{l^{\prime}}\left(V_{1}\right)=\emptyset$ when $l \neq l^{\prime}$, therefore we have volume $\left.\left(\cup_{0}^{k} \mathcal{N}_{2}\left(\partial N_{k}\right)\right)\right) \geq 4 k^{2} \operatorname{volume}\left(V_{1}\right)$. But, by hypothesis we have that volume $\left.\left.\left(\cup_{0}^{k} \mathcal{N}_{2}\left(\partial N_{k}\right)\right)\right) \leq \sum_{0}^{k} \operatorname{volume}\left(\mathcal{N}_{2}\left(\partial N_{k}\right)\right)\right) \leq(k+1) C$, for some constant $C>0$. Hence, there exists a constant $C^{\prime}>0$ such that we have $4 k^{2} \leq k C^{\prime}$ for all $k$, which is a contradiction. So, we have a dense subset $U$ of $N$ with $\left.\operatorname{grad} u\right|_{U}=0$. By continuity we get $\left.\operatorname{grad} u\right|_{N}=0$, which implies $u$ is constant on $N$.

COROLlaRy 4.6. Take $N$ as in Theorem 4.5. Then there exist no nontrivial positive superharmonic functions on $N$. 
COROLlary 4.7. Let $M=\tilde{M} / \Gamma$ be a topologically tame negatively pinched $\left(-b^{2} \leq \mathcal{K} \leq-a^{2}\right) 3$-manifold with $\Lambda(\Gamma)=S_{\infty}$. Then there are no nonconstant positive superharmonic functions, or nonconstant subharmonic functions bounded above, on $M$.

Proof. By hypothesis, we have $\Lambda(\Gamma)=S_{\infty}$ which implies $\mathcal{C C}(M)=M$. Then the result follows from Corollary 4.4 and Theorem 4.5 and Corollary 4.6.

5. $\Gamma$-action. Two Borel measures on $S_{\infty}$ are in the same $\Gamma$-class if the RadonNikodym derivative of $\gamma^{*} v_{1}$ with respect to $v_{1}$ is equal to the Radon-Nikodym derivative of $\gamma^{*} v_{2}$ with respect to $v_{2}$.

Proposition 5.1 ([29]). Let $\Gamma$ be nonelementary and discrete. Suppose that $\Gamma$ acts ergodically on $S_{\infty}$ with respect to a measure $v$ defined on $S_{\infty}$. Then every measure of $S_{\infty}$ in the same measure class as $v$ is a constant multiple of $v$.

A family of finite Borel measures $\left[v_{y}\right]_{y \in \tilde{M}}$ will be called a $\lambda$-(conformal density under the action of $\Gamma$ ) if for every $x \in \tilde{M}$ and every $\gamma \in \Gamma$ we have $\gamma^{*} v_{y}=v_{\gamma^{*} y}$, and the RadonNikodym derivative $\frac{\mathrm{d} v_{y}}{\mathrm{~d} \gamma^{*} v_{y}}(\zeta)$ at any point $\zeta \in S_{\infty}$ is equal to $\exp \left(-\lambda B_{\zeta}\left(\gamma^{-1} y, y\right)\right)$. (This is to be interpreted as being vacuously true if, for example, the measures in the family are all identically zero.)

Proposition 5.2. Let $\Gamma$ be a non-elementary discrete subgroup of the isometry group of $\tilde{M}$. If $\left[v_{y}\right]_{y \in \tilde{M}}^{D}$ is a non-trivial $\Gamma$-invariant $D$-conformal density, then $D \neq 0$.

Proof. Suppose $D=0$. Then $v_{y}$ is a $\Gamma$-invariant non-trivial finite Borel measure. Since $\Gamma$ is non-elementary, there exists a loxodromic element $\gamma$ in $\Gamma$. Let $\xi, \zeta \in S_{\infty}$ be the two distinct fixed points of $\gamma$. Let $\langle\gamma\rangle$ be the group generated by $\gamma$. Then $v_{y}$ is clearly $\langle\gamma\rangle$-invariant. But $\gamma$ is loxodromic, so we must have $\operatorname{supp}\left(v_{y}\right) \subset\{\xi, \zeta\}$. Then, by the fact that $\Lambda(\Gamma)$ is infinite, we have $v_{y}$ is an infinite measure, which is a contradiction.

Let $x \in \tilde{M}$ and $s>0$ be given. Denote the Poincare series for a infinite uniformly discrete subset $W \subset \tilde{M}$ by $Z_{W}(x, s)$, i.e. set

$$
Z_{W}(x, s):=\sum_{v \in W} \exp (-s \operatorname{dist}(x, v)) .
$$

In particular, $W$ can be the orbit $\Gamma x$ of $x$. We will use the notation $Z_{\Gamma}(x)$ to denote the Poincare series for $W=\Gamma x$ in this case. We call $\Gamma$ divergent if $Z_{\Gamma}=\infty$. By applying an adjusting function we can always assume the Poincaré series diverges at $D$. The measures

$$
\mu_{x, s}:=\frac{\sum_{\gamma \in \Gamma} e^{-s \operatorname{dist}(z, \gamma x)} \delta_{\gamma x}}{\sum_{\gamma \in \Gamma} e^{-s \operatorname{dist}(z, \gamma z)}} ; s>D
$$

converges weakly to a limiting measure $\mu_{x}$ as $s_{n} \rightarrow D$ through a subsequence. It is trivial to see that $\mu_{x}$ is supported on $\Lambda_{\Gamma}$. The measure $\left[\mu_{x}\right]^{D}$ is called PattersonSullivan measure which is $D$-conformal under $\Gamma$.

TheOREM 5.3 (Hou [24]). Let $M=\tilde{M} / \Gamma$ be a complete Riemannian 3-manifold with $-b^{2} \leq \mathcal{K} \leq-1$, such that there are no nontrivial positive superharmonic functions 
on $M$. Then $D \in[2,2 b]$. And if we have $D=2$, then $\Gamma$ is divergent, hence ergodic with respect to $\left[\mu_{x}\right]^{D}$.

Proof of Theorem 1.2. Under the hypothesis of Theorem 1.2, it follows from Corollary 4.7 and Theorem 5.3 that $D \in[2,2 b]$. That $\Gamma$ is harmonically ergodic follows from Corollary 4.7 and Fatou's conical convergence theorem. If $D=2$, then, by Corollary 4.7 and Theorem 5.3, we have $\Gamma$ is divergent.

ACKNOWledgements. I wish to think my advisor Peter Shalen for his constant advice and encouragement. I like to dedicate this paper to Qun Hou and Chun Xue Hou for their inspiration and continuous support.

\section{REFERENCES}

1. W. Ballman, M. Gromov and V. Schroeder, Manifolds of nonpositive curvature (Birkhäuser, 1985).

2. R. Benedetti and C. Petronio, Lecture on hyperbolic geometry (Springer, Berlin, 1992).

3. A. Besse, Einstein manifolds (Springer-Verlag, 1986).

4. G. Besson, G. Courtois and S. Gallot, Entropies et rigidités des espaces localement symétriques de courbure strictement négative, Geom. Funct. Anal. 5 (1995), 731-799. $71-158$.

5. F. Bonahon, Bouts des variétés hyperboliques de dimension 3. Ann. Math. 124 (1986),

6. F. Bonahon, Cobordism of automorphisms of surfaces, Ann. Sci. École. Norm. Sup. 16 (1983), 237-270.

7. B. Bowditch, Geometrical finiteness with variable negative curvature, Duke. Math. J. 77 (1995), 229-274.

8. R. Canary, Ends of hyperbolic 3-manifolds, J. Amer. Math. Soc. 6 (1993), 1-35.

9. R. Canary, A covering theorem for hyperbolic 3-manifolds and its applications, Topology 35 (1996), 751-778.

10. R. Canary, D. Epstein and P. Green, (1987), Notes on notes of Thurston, Analytical and geometric aspects of hyperbolic space, London Math. Soc. Lecture Notes. 112 (Cambridge University Press, 1987), 3-92.

11. M. Coornaert, Sur les groups proprement discontinus d'isometriés des espaces hyperboliques au sens de Gromov. Thése, 1990.

12. M. Culler, S. Hersonsky and B. P. Shalen, The first Betti number of the smallest closed hyperbolic 3-manifolds, Topology 37 (1998), 805-849.

13. J. Cheeger, (1990), Critical points of distance functions and applications to geometry, Lecture Notes at NYU.

14. M. Feighn and D. McCullough, Finiteness conditions for 3-manifolds with boundary, Amer. J. Math. 109 (1987), 1155-1169.

15. P. Eberlin and B. O'Neil, Visibility manifolds, Pacific. J. Math. 46 (1973), 45-109.

16. D. Ebin, The manifolds of Riemannian metrics, Global Analysis, (ed. S. S. Chern and S. Smale) Proc. Symp. Pure. Math. 15 (Amer. Math. Soc., Providence, 1970).

17. L. Z. Gao, The construction of negative Ricci curved manifolds, Math. Ann. 271 (1985), $185-208$

18. L. Z. Gao and S. T. Yau, The existence of negatively Ricci curved metrics on three manifolds, Invent. Math. 85 (1988), 637-652.

19. M. Gromov, Hyperbolic groups, in Essays in group theory, Math. Sci. Res. Inst. Publ. 8 (Springer-Verlag, 1987), 75-263.

20. M. Gromov and W. Thurston, Pinching constants for hyperbolic manifolds, Invent. Math. 89 (1987), 1-12.

21. E. Heintze and H. C. Im Hof, On the geometry of horospheres, J. Diff. Geom. 12 (1977), $481-491$.

22. J. Hempel, (1976). 3-manifolds, Ann. Math. Studies. 86, Princeton Univ. Press. 
23. Y. Hou, Critical exponent and displacement of negatively curved free groups, J. Diff. Geom. 57 (2001), 173-195.

24. Y. Hou, Quasiconformal rigidity of negatively curved three-manifolds, Asian. J. Math., to appear.

25. W. Jaco and P. Shalen, Seifert fibered spaces in 3-manifolds, Mem. Amer. Math. Soc. 21 (1979).

26. V. A. Kaimanovich, Invariant measures for the geodesic flow and measures at infinity on negatively curved manifolds, Ann. Inst. Henri Poincaré, Phys. Théor. 53 (1990), No 4, 361-393.

27. W. Meeks and S. T. Yau, The equivariant Dehn's lemma and loop theorem, Comm. Math. Helv. 56, 225-239.

28. J. Morgan, On Thurston's uniformization theorem for three-dimensional manifolds, The Smith Conjecture (ed. J. Morgan and H. Bass) (Academic Press, New York, 1984), 37-125.

29. P. J. Nicholls, The ergodic theory of discrete groups (Cambridge University Press, 1989).

30. J.-P. Otal, Thurston's hyperbolization of Haken manifolds, Surveys in differential geometry, Vol. III (Inter. Press, Boston, 1998), 77-194.

31. S. J. Patterson, Lectures on measures on limit sets of Kleinian groups, Analytical and geometrical aspects of hyperbolic space, London Math. Soc. Lecture Notes Series III (Cambridge University Press 1987), 291-323.

32. D. Sullivan, Discrete conformal groups and measurable dynamics, Bull. Amer. Math. Soc. 6 (1982), 57-73.

33. D. Sullivan, The density at infinity of a discrete group of hyperbolic motions, Publ. Math. I.H.E.S. 50 (1979), 171-202.

34. D. Sullivan, Related aspects of positivity in Riemannian geometry, J. Diff. Geom. 25 (1987), 327-351. Univ.

35. W. Thurston, The geometry and topology of 3-manifolds. Lecture notes, Princeton

36. F. Waldhausen, On irreducible 3-manifolds which are sufficiently large, Ann. Math. 87 (1968), 56-88. 